\title{
New Valid Inequalities and Facets for the Simple Plant Location Problem
}

\author{
Laura Galli* Adam N. Letchford ${ }^{\dagger} \quad$ Sebastian J. Miller ${ }^{\ddagger}$ \\ To appear in European Journal of Operational Research
}

\begin{abstract}
The Simple Plant Location Problem is a well-known (and $\mathcal{N} \mathcal{P}$-hard) combinatorial optimisation problem, with applications in logistics. We present a new family of valid inequalities for the associated family of polyhedra, and show that it contains an exponentially large number of new facet-defining members. We also present a new procedure, called facility augmentation, which enables one to derive even more valid and facet-defining inequalities.
\end{abstract}

Keywords: facility location; combinatorial optimisation; branch-andcut; polyhedral combinatorics

\section{Introduction}

The Simple Plant Location Problem (SPLP), sometimes called the Uncapacitated Facility Location Problem, is a much-studied problem in Operational Research. We are given a set $I$ of facilities and a set $J$ of clients. The cost of opening facility $i \in I$ is denoted by $f_{i}$, and the cost of assigning client $j \in J$ to facility $i \in I$ is denoted by $c_{i j}$. The task is to decide which facilities to open, and then to assign each client to an open facility, at minimum cost.

Balinski [2] showed that the set covering problem can be transformed to the SPLP, which implies that the SPLP is $\mathcal{N} \mathcal{P}$-hard in the strong sense. Surveys on theory, algorithms and applications of the SPLP include [10, 12, $19,23]$. The current leading exact algorithms, such as those in $[20,21]$, are capable of solving random planar instances with thousands of facilities and clients to proven optimality. There are however much smaller instances, such as the ones in $[14,16,18]$, that still remain a challenge for exact methods.

\footnotetext{
*Dipartimento di Informatica, Università di Pisa, Largo B. Pontecorvo 3, 56127 Pisa, Italy. E-mail: Laura.Galli@di.unipi.it

${ }^{\dagger}$ Department of Management Science, Lancaster University, Lancaster LA1 4YX, United Kingdom. E-mail: A.N.Letchford@lancaster.ac.uk

${ }^{\ddagger}$ Former PhD student at Lancaster University. E-mail: sebmiller64@hotmail.co.uk
} 
Here, we are concerned with the integer programming approach to the SPLP, and in particular on valid and facet-defining inequalities for the associated polyhedra. We assume throughout that the reader is familiar with the polyhedral approach to combinatorial optimisation. Readers who are not are referred to standard textbooks, such as $[8,17]$.

Balinski [2] formulated the SPLP as follows:

$$
\begin{array}{ccl}
\min & \sum_{i \in I} \sum_{j \in J} c_{i j} x_{i j}+\sum_{i \in I} f_{i} y_{i} & \\
\mathrm{s.t.} & \sum_{i \in I} x_{i j}=1 & (j \in J) \\
& x_{i j} \leq y_{i} & (i \in I, j \in J) \\
& x_{i j} \in\{0,1\} & (i \in I, j \in J) \\
& y_{i} \in\{0,1\} & (i \in I) .
\end{array}
$$

Here, $y_{i}$ is a binary variable, taking the value 1 if and only if a facility $i$ is opened, and $x_{i j}$ is a binary variable, taking the value 1 if and only if client $j$ is assigned to facility $i$. The constraints (2) are called assignment constraints, and the constraints (3) are called variable upper bounds (VUBs).

The polyhedra associated with the formulation (1)-(5) have been studied in depth, and several families of valid and facet-defining inequalities are known $[1,3,4,5,6,9,11,15]$. The main purpose of this paper is to introduce some new, exponentially large, families of valid and facet-defining inequalities, along with a new lifting procedure. A secondary purpose is to shed new light on some of the known families of inequalities.

The paper is structured as follows. The literature is reviewed in Sect. 2. In Sect. 3, we introduce the homogeneous inequalities (HIs) and show that they subsume the combinatorial inequalities given in $[5,6]$. In Sect. 4, we revisit two known families of inequalities, first presented in [11] and [1], and prove some new results about them. In Sect. 5, we present the new lifting procedure. Some concluding remarks are given in Sect. 6. The design of effective separation heuristics for the new inequalities (and some known ones) will be the subject of a future paper.

The following notation is used throughout the paper. We let $m$ denote $|I|$ and $n$ denote $|J|$. We let $P(m, n)$ denote the SPLP polytope, i.e., the convex hull of all pairs $(x, y) \in \mathbb{R}^{(m \times n)+m}$ that satisfy the constraints (2)-(5). To avoid using subscripted subscripts, we sometimes write $x(i, j)$ instead of $x_{i j}$, and $y(i)$ instead of $y_{i}$. Moreover, sometimes we write $x(E)$ for $\sum_{\{i, j\} \in E} x_{i j}$, $x(S: T)$ for $\sum_{i \in S} \sum_{j \in T} x_{i j}$ and $y(S)$ for $\sum_{i \in S} y_{i}$.

\section{Literature Review}

Since the SPLP literature is vast, we focus here on papers that present polyhedral results, and refer the reader to $[10,12,19,23]$ for surveys of other approaches. 


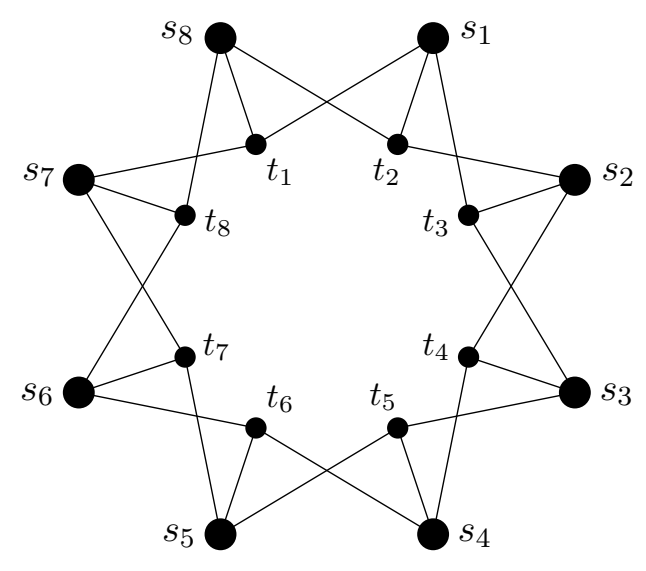

Figure 1: Graph corresponding to a circulant inequality with $p=8$ and $q=3$.

\subsection{Dimension and trivial facets}

Cornuéjols \& Thizy [11] showed that the dimension of $P(m, n)$ is $m n+m-n$, and that the affine hull is described by the assignment constraints (2). It is also shown that the VUBs (3), the non-negativity constraints $x_{i j} \geq 0$ for all $i$ and $j$, and the upper bounds $y_{i} \leq 1$ for all $i$ define trivial facets of $P(m, n)$.

\subsection{Circulant and odd cycle inequalities}

Cornuéjols et al. [9] showed the following. Let $p$ and $q$ be integers satisfying $2 \leq q<p \leq m$ and $p \leq n$, with $p$ not a multiple of $q$. Let $s_{1}, \ldots, s_{p}$ be distinct facilities, let $t_{1}, \ldots, t_{p}$ be distinct clients, and take indices modulo $p$, so that, for example, $s_{p+1}$ is identified with $s_{1}$. Then, the inequality

$$
\sum_{i=1}^{p} \sum_{j=i}^{i+q-1} x\left(s_{i}, t_{j}\right) \leq \sum_{i=1}^{p} y\left(s_{i}\right)+p-\lceil p / q\rceil
$$

is valid for $P(m, n)$.

We call the inequalities (6) circulant inequalities. Figure 1 represents a circulant inequality with $p=8$ and $q=3$. The large and small circles represent facilities and clients, respectively, and the edges represent variables that appear on the left-hand side. Guignard [15] showed that circulant inequalities with $p=q+1$, which we call simple, define facets. Cornuéjols \& Thizy [11] showed that non-simple circulant inequalities do not.

The circulant inequalities with $q=2$ (and therefore $p$ odd) are called odd cycle inequalities $[5,11]$. The only odd cycle inequalities that define facets are those with $p=3$, which we call 3 -cycle inequalities. 


\subsection{Combinatorial and $(p, q)$ inequalities}

It is easy to show that all non-trivial facets of $P(m, n)$ are defined by inequalities of the form $\alpha^{T} x \leq \beta^{T} y+\gamma$, with $\alpha$ and $\beta$ being non-negative integer vectors and $\gamma$ being a positive integer. Cho et al. $[5,6]$ studied the inequalities in which both $\alpha$ and $\beta$ have binary components. We follow Aardal [1] in calling them combinatorial inequalities. Associated with any combinatorial inequality is a bipartite graph $G=\left(I^{\prime}, J^{\prime}, E\right)$, where $I^{\prime}$ is the set of facilities such that $\beta_{i}=1, J^{\prime}$ is the set of clients for which at least one $\alpha_{i j}$ takes the value 1 , and $E$ is the set of pairs $\{i, j\}$ such that $\alpha_{i j}=1$. Cho et al. [5] showed that a combinatorial inequality is supporting (i.e., defines a non-empty face of $P(m, n))$ if and only if $\gamma=\left|J^{\prime}\right|-\kappa(G)$, where

$$
\kappa(G)=\min \left\{y\left(I^{\prime}\right):(2)-(5), x(E)=\left|J^{\prime}\right|\right\}
$$

is the so-called covering number of $G$. Cho et al. [6] proved that a combinatorial inequality defines a facet if and only if (a) it is supporting, (b) $\beta$ has at least three non-zero components, and (c) the inequality cannot be lifted by changing some component of $\alpha$ from 0 to 1 .

Unfortunately, computing $\kappa(G)$ is $\mathcal{N} \mathcal{P}$-hard (by reduction from set cover), and it seems likely that checking whether a combinatorial inequality can be lifted is co- $\mathcal{N} \mathcal{P}$-complete. Some explicit examples of facet-defining combinatorial inequalities (besides the simple circulant inequalities mentioned above) are given in $[4,5,6,11]$.

Aardal [1] considered a family of valid inequalities that are intermediate in generality between the circulant and combinatorial inequalities. Let $p$ and $q$ be integers satisfying $2 \leq q<p \leq n$, with $p$ not a multiple of $q$. Let $I^{\prime} \subseteq I$ be any facility set with $\left|I^{\prime}\right| \geq\lceil p / q\rceil$ and $J^{\prime} \subseteq J$ be any client set with $\left|J^{\prime}\right|=p$. Let $G$ be any bipartite graph with node sets $I^{\prime}$ and $J^{\prime}$, such that each node in $I^{\prime}$ has degree $q$ in $G$. Finally, let $E$ denote the set of edges of $G$. Then the inequality

$$
x(E) \leq y\left(I^{\prime}\right)+p-\lceil p / q\rceil
$$

is valid. We call these inequalities $(p, q)$ inequalities. Aardal does not discuss conditions for them to define facets. We remark that $\lceil p / q\rceil$ is only a lower bound on the covering number, and therefore $(p, q)$ inequalities are not guaranteed to be supporting in general.

\subsection{Other inequalities}

Some non-combinatorial facet-defining inequalities are also known. Cho et al. [6] presented a family of inequalities in which $\beta$ is binary, $\gamma=2$, and the components of $\alpha$ belong to $\{0,1,2\}$. Cánovas et al. [3] introduce the so-called grille inequalities, in which $\alpha$ is binary and $\gamma=1$, but $\beta$ can be arbitrary. Cánovas et al. [4] showed that the grille inequalities are the 
only facet-defining inequalities of that type, and also introduced some other non-combinatorial inequalities, called fan and wheel inequalities. We skip details, for the sake of brevity.

\section{Homogeneous Inequalities}

In this section, we examine what we call "homogenous" inequalities. These are defined as follows.

Definition $1 A$ valid inequality for $P(m, n)$ is homogeneous if there exist sets $I^{\prime} \subseteq I$ and $J^{\prime} \subseteq J$ and a connected bipartite graph $G=\left(I^{\prime}, J^{\prime}, E\right)$ such that the inequality can be written as:

$$
\alpha x(E) \leq \beta y\left(I^{\prime}\right)+\gamma
$$

for some relatively prime positive integers $\alpha, \beta$ and some integer $\gamma$.

We will often call homogeneous inequalities HIs for short. Note that combinatorial inequalities are nothing but HIs with $\alpha=\beta=1$.

In Subsect. 3.1, we define and analyse what we call strong HIs. In Subsect. 3.2, we define and analyse what we call simple HIs.

\subsection{Strong homogeneous inequalities}

Let us say that an HI is strong if it is not implied by other HIs involving the same graph $G$. (Note that being strong is a necessary but not sufficient condition for an HI to define a facet.) The following definition, lemma and proposition show how to compute all strong HIs for a given $G$.

Definition 2 For any given graph $G=\left(I^{\prime}, J^{\prime}, E\right)$, let $\kappa(G)$ be the covering number and, for $k=0, \ldots, \kappa(G)$, define

$$
\Phi_{G}(k)=\max \left\{x(E):(2)-(5), y\left(I^{\prime}\right)=k\right\} .
$$

Finally, for $k=1, \ldots, \kappa(G)$, define $\Delta_{G}(k)=\Phi_{G}(k)-\Phi_{G}(k-1)$.

We remark that $\Phi_{G}(0)=0$ and $\Phi_{G}(\kappa(G))=\left|J^{\prime}\right|$.

Lemma 1 For any $G$, the function $\Phi_{G}$ is subadditive.

Proof. Suppose that $k_{1}+k_{2}=k$. Consider an SPLP solution in which $y\left(I^{\prime}\right)=k$ and $x(E)=\phi_{G}(k)$. Suppose w.l.o.g. that the open facilities are $1, \ldots, k$ and that they are sorted in non-increasing order of $\sum_{j \in J^{\prime}} x_{i j}$. If we keep the first $k_{1}$ facilities open and close the rest, we obtain an SPLP solution in which $y\left(I^{\prime}\right)=k_{1}$ and $x(E) \geq\left(k_{1} / k\right) \Phi_{G}(k)$. Thus, $\Phi_{G}\left(k_{1}\right) \geq$ $\left(k_{1} / k\right) \Phi_{G}(k)$. For the same reason, $\Phi_{G}\left(k_{2}\right) \geq\left(k_{2} / k\right) \Phi_{G}(k)$. So $\Phi_{G}(k)=$ $\left(k_{1} / k\right) \Phi_{G}(k)+\left(k_{2} / k\right) \Phi_{G}(k) \leq \Phi_{G}\left(k_{1}\right)+\Phi_{G}\left(k_{2}\right)$. 
Proposition 1 For any $G$, the strong HIs take the form

$$
x(E) \leq \Delta_{G}(k) y\left(I^{\prime}\right)+\Phi_{G}(k)-k \Delta_{G}(k)
$$

for some $k \in\{1, \ldots, \kappa(G)\}$. (In particular, all strong HIs have $\alpha=1$.)

Proof. Let $P_{2}$ denote the projection of $P(m, n)$ into a 2-dimensional subspace with $y\left(I^{\prime}\right)$ and $x(E)$ as axes. That is,

$$
P_{2}=\left\{z \in \mathbb{R}^{2}: \exists(\bar{x}, \bar{y}) \in P(m, n): z_{1}=\bar{y}\left(I^{\prime}\right), z_{2}=\bar{x}(E)\right\} .
$$

By definition, when $I^{\prime} \neq I$, we have

$$
P_{2}=\operatorname{conv}\left\{z \in \mathbb{Z}_{+}^{2}: z_{1} \leq\left|I^{\prime}\right|, z_{2} \leq \Phi_{G}\left(z_{1}\right)\right\} .
$$

(When $I^{\prime}=I$, we also have to add the trivial inequality $z_{1} \geq 1$, since every feasible SPLP solution has at least one open facility.) Since $\Phi_{G}$ is subadditive, every point with $y\left(I^{\prime}\right)=k$ and $x(E)=\Phi(k)$ for some $k$ projects to a point on the boundary of $P_{2}$. One can check that the HI (8) is satisfied at equality by two consecutive points on the boundary, and therefore defines a facet of $P_{2}$.

Fig. 2 illustrates Definition 2 and Proposition 1 for the $(8,3)$-circulant, which was shown in Fig. 1 . We have $\kappa(G)=3, \Phi_{G}(1)=3, \Phi_{G}(2)=6$, $\Delta_{G}(1)=\Delta_{G}(2)=3$ and $\Delta_{G}(3)=2$. The resulting strong HIs, represented by dashed lines in the figure, are $x(E) \leq 3 y\left(I^{\prime}\right)$ and $x(E) \leq 2 y\left(I^{\prime}\right)+2$. The first is redundant, being implied by the VUBs for the edges in $E$, but the second is non-redundant. (Indeed, the inequality $x(E) \leq 2 y\left(I^{\prime}\right)+2$, together with the trivial inequality $x(E) \leq 8$, dominates the $(8,3)$-circulant inequality, which is $x(E) \leq y\left(I^{\prime}\right)+5$.)

The following result is more or less immediate.

Proposition 2 Every combinatorial inequality is either equivalent to or dominated by a strong HI with $k=\kappa(G)$.

Proof. Recall (from Subsection 2.3) that the supporting combinatorial inequalities take the form $x(E) \leq y\left(I^{\prime}\right)+\left|J^{\prime}\right|-\kappa(G)$. The corresponding strong HI with $k=\kappa(G)$ takes the form

$$
x(E) \leq \Delta_{G}(\kappa(G)) y\left(I^{\prime}\right)+\left|J^{\prime}\right|-\Delta_{G}(\kappa(G)) \kappa(G) .
$$

One can check that this HI, together with the trivial inequality $x(E) \leq\left|J^{\prime}\right|$, implies the given combinatorial inequality. Moreover, the HI is equivalent to the combinatorial inequality if and only if $\Delta_{G}(\kappa(G))=1$.

Another positive result concerned with strong HIs is the following: 


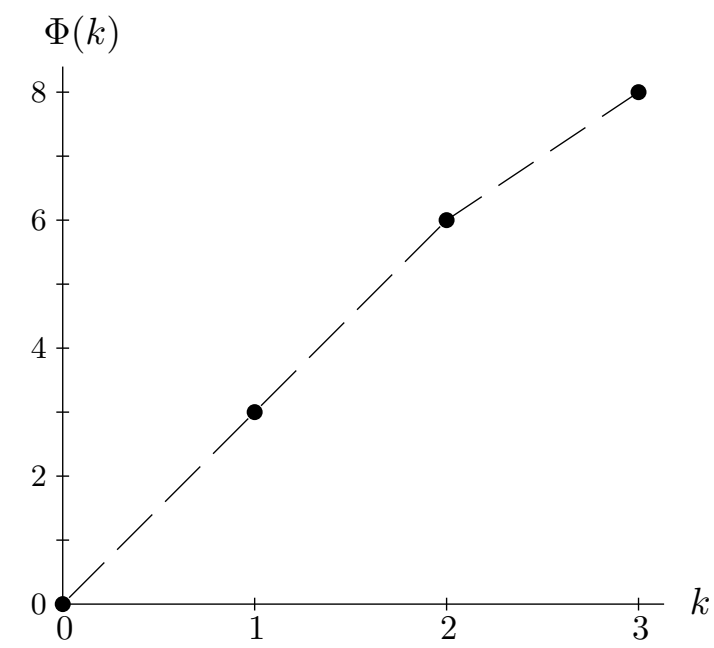

Figure 2: Sketch of the function $\Phi_{G}(k)$ for the graph shown in Fig. 1.

Theorem 1 There exist facet-defining strong HIs which are neither combinatorial inequalities nor grille inequalities. Moreover, there exist inequalities of this type with $k<\kappa(G)$.

Proof. See Appendix A.

A natural question at this point is, under which conditions do strong HIs define facets? The following proposition gives some conditions that the graph $G$ must satisfy.

Proposition 3 Suppose that $G$ is given. Let $J(i)$ denote the set of nodes adjacent to node $i \in I^{\prime}$, and $I(j)$ denote the set of nodes adjacent to node $j \in J^{\prime}$. If a strong $H I$ for the given $G$ defines a facet of $P(m, n)$, then:

1. $\left|I^{\prime}\right| \geq 3$ and $\left|J^{\prime}\right| \geq 3$;

2. $2 \leq|J(i)|<\left|J^{\prime}\right|$ for all $i \in I^{\prime}$ and $2 \leq|I(j)|<\left|I^{\prime}\right|$ for all $j \in J^{\prime}$;

3. there is no pair $i, i^{\prime} \in I^{\prime}$ such that $J(i)$ is a proper subset of $J\left(i^{\prime}\right)$;

4. $G$ is biconnected (i.e., has no cut nodes);

5. there is no edge $\{i, j\} \in E$ such that the removal of $i$ and $j$ disconnects $G$;

Proof. For the sake of brevity, we only sketch the proof. Point 1 follows from Remark 2.3 in [5]. The lower bounds of 2 on $|J(i)|$ and $|I(j)|$ in Point 2 are in Theorem 5.1 in [5]. If $J(i)=J^{\prime}$ for some $i \in I^{\prime}$, we have 
$\Phi_{G}(1)=\Delta_{G}(1)=\left|J^{\prime}\right|$, and the HI reduces to $x(E) \leq\left|J^{\prime}\right| y\left(I^{\prime}\right)$, which is implied by the VUBs for the edges in $E$. If $I(j)=I^{\prime}$ for some $j \in J^{\prime}$, the HI can be strengthened by dropping $j$ from $J^{\prime}$. For Point 3 , if there is a pair $i, i^{\prime} \in I^{\prime}$ such that $J(i)$ is a proper subset of $J\left(i^{\prime}\right)$, then the HI can be strengthened by adding edges to $E$ so that $J(i)=J\left(i^{\prime}\right)$. If the conditions in Points 4 or 5 are not satisfied, then the intersection graph (the graph with node set $I^{\prime} \cup E$ and an edge between $i$ and $\{i, j\}$ for all $\{i, j\} \in E$ ) contains a clique cutset, i.e., a clique whose removal disconnects the graph. Points 4 and 5 then follow from Theorem 4.1 in Chvátal [7].

Unlike in the case of the combinatorial inequalities, we have to consider not only $G$, but also the parameter $k$.

Proposition 4 If the strong HI for a given pair $(G, k)$ defines a facet of $P(m, n)$, then:

1. $k \geq 2$;

2. $\beta=\Delta_{G}(k)<\max _{i \in I^{\prime}}|J(i)|$;

3. $G$ is maximal for the given $k$; i.e., adding any edge to $G$ would cause the HI to become invalid.

Proof. For any $G$, we have $\Phi_{G}(1)=\Delta_{G}(1)=\max _{i \in I^{\prime}}|J(i)|$. So the strong HI for $k=1$ reduces to

$$
x(E) \leq \max _{j \in J^{\prime}}|I(j)| y\left(I^{\prime}\right),
$$

which is implied by the VUBs. Since $\Phi_{G}$ is subadditive, the $\Delta_{G}(k)$ values cannot increase as $k$ increases. This implies that $\Delta_{G}(k) \leq \max _{j \in J^{\prime}}|I(j)|$ for $k \geq 2$. If $\Delta_{G}(k)=\max _{j \in J^{\prime}}|I(j)|$ for some $k \geq 2$, then the strong HI again reduces to the redundant inequality (9). Finally, if $G$ were not maximal, we could obtain a stronger HI by adding an edge to $E$.

Unfortunately, the conditions in the above two propositions are not sufficient:

Proposition 5 There exist strong HIs that satisfy all of the conditions listed in Propositions 3 and 4, and cut off fractional LP solutions, yet do not define facets.

Proof. Let $G$ be the $(8,3)$-circulant, which was shown in Fig. 1. Above, we showed that the strong HI $x(E) \leq 2 y\left(I^{\prime}\right)+2$ is valid. One can check that this strong HI satisfies the conditions in Proposition 3 , and that it cuts off fractional solutions with $x_{i j}=1 / 3$ for all $\{i, j\} \in E$ and $y_{i}=1 / 3$ for all $i \in I^{\prime}$. Despite this, it does not define a facet, since every SPLP solution satisfying it at equality also satisfies the equation $x\left(s_{1}, t_{6}\right)=0$.

In this sense, the strong HIs are even less "well-behaved" than the combinatorial inequalities. 


\subsection{Simple homogeneous inequalities}

Recall (from Subsection 2.3) that computing $\kappa(G)$ is $\mathcal{N} \mathcal{P}$-hard in the strong sense. It seems likely that computing the $\Phi_{G}(k)$ is also hard. The following proposition introduces some HIs that may not be strong, but whose coefficients can be computed efficiently.

Proposition 6 Given a graph $G$ as before, define

$$
\kappa^{-}(G)=\left\lceil\min \left\{y\left(I^{\prime}\right):(2),(3), x(E)=\left|J^{\prime}\right|, x \in[0,1]^{|I||J|}, y \in[0,1]^{|I|}\right\}\right\rceil .
$$

For $k=0, \ldots, \kappa^{-}(G)$, define

$$
\Phi_{G}^{+}(k)=\left\lfloor\max \left\{x(E):(2),(3), y\left(I^{\prime}\right)=k, x \in[0,1]^{|I||J|}, y \in[0,1]^{|I|}\right\}\right\rfloor .
$$

Finally, for $k=1, \ldots, \kappa^{-}(G)$, define $\tilde{\Delta}_{G}(k)=\Phi_{G}^{+}(k)-\Phi_{G}^{+}(k-1)$. Then for any $k \in\left\{1, \ldots, \kappa^{-}(G)\right\}$, the inequality

$$
x(E) \leq \tilde{\Delta}_{G}(k) y\left(I^{\prime}\right)+\Phi_{G}^{+}(k)-k \tilde{\Delta}_{G}(k)
$$

is valid for $P(m, n)$.

Proof. By definition, $\kappa^{-}(G)$ is a lower bound on $\kappa(G)$, and $\Phi_{G}^{+}(k)$ is an upper bound on $\Phi_{G}(k)$. The rest of the proof is similar to that of Lemma 1 and Proposition 1.

We call the HIs (10) simple. By definition, every simple HI is equivalent to or dominated by a strong HI. On the other hand, the advantage of the simple HIs is that their coefficients can be computed in polynomial time (since the values $\kappa^{-}(G)$ and $\Phi_{G}^{+}(k)$ are found by solving linear programs). We also have the following proposition, which is analogous to Proposition 2.

Proposition 7 Every $(p, q)$ inequality is equivalent to or dominated by a simple HI with $k=\kappa^{-}(G)$.

Proof. Consider an arbitrary $(p, q)$ inequality (7), with associated graph $G=(S \cup T, E)$. We have $\kappa^{-}(G) \geq\lceil p / q\rceil, \Phi_{G}^{+}(\lceil p / q\rceil) \leq p$ and $\Phi_{G}^{+}(\lceil p / q\rceil) \leq$ $p-1$, with equality holding if and only if the simple HI with $k=\kappa^{-}(G)$ is a $(p, q)$ inequality.

We now show that there exist facet-defining simple HIs that are not equivalent to previously known inequalities.

Theorem 2 Let $p$ and $q$ be positive integers with $2 \leq q<p \leq n, m \geq\left(\begin{array}{l}p \\ q\end{array}\right)$ and $p$ not a multiple of $p$. Let $S$ be a set of facilities such that $|S|=\left(\begin{array}{l}p \\ q\end{array}\right)$, and let $T$ be a set of clients such that $|T|=p$. Let $G$ be any bipartite graph with 


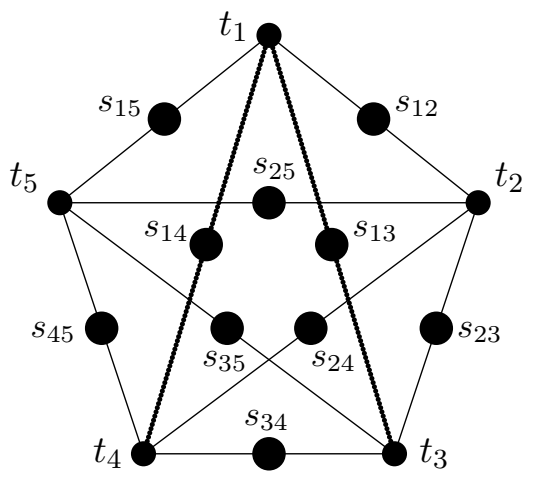

Figure 3: Graph $G$ corresponding to a facet-defining simple HI with $p=5$ and $q=2$.

node sets $S$ and $T$, such that, for every set $T^{\prime} \subset T$ with $\left|T^{\prime}\right|=q$, there exists an $i \in S$ that is connected to each node in $T^{\prime}$ and no other nodes. (Figure 3 shows a suitable graph $G$ for the case $p=5, q=2$. The five nodes in $T$ are labelled $t_{1}, \ldots, t_{5}$ and the ten nodes in $S$ are labelled $\left.s_{12}, \ldots, s_{45}.\right)$ Finally, let $E$ denote the edge set of $G$. The simple $H I$ with $k=\kappa^{-}(G)$ defines a facet of $P(m, n)$.

Proof. See Appendix B.

Note that the number of facet-defining simple HIs described in Theorem 2 grows exponentially with both $m$ and $n$. We leave open the question of finding a necessary and sufficient condition for a simple HI to define a facet. We also leave open the question of whether there exist facet-defining simple HIs with $k<\kappa^{-}(G)$.

\section{Circulant and $(p, q)$ Inequalities Revisited}

In this section, we use the results in the previous section to shed new light on some of the inequalities in the literature. We cover circulant inequalities in Subsect. 4.1 and $(p, q)$ inequalities in Subsect. 4.2. Throughout this section, we use the notation $p=w q+r$, as in the proof of Theorem 2 .

\subsection{On circulant inequalities}

Consider once more the circulant inequalities (6). One can check that, regardless of the values of $p$ and $q$, we have $\kappa(G)=\kappa^{-}(G)=w+1, \Phi_{G}(w)=$ $\Phi_{G}^{+}(w)=w q, \Phi_{G}(w+1)=\Phi_{G}^{+}(w+1)=p$ and $\Delta_{G}(w+1)=\tilde{\Delta}_{G}(w+1)=r$. Together with Proposition 1 (or Proposition 6), this yields the following result. 
Corollary 1 Every circulant inequality is equivalent to or dominated by an HI of the form

$$
\sum_{i=1}^{p} \sum_{j=i}^{i+q} x\left(s_{i}, t_{j}\right) \leq r \sum_{i=1}^{p} y\left(s_{i}\right)+w(q-r) .
$$

This HI is both strong and simple.

We call the inequalities (11) strengthened circulant inequalities or SCIs. One can check that they are equivalent to circulant inequalities when $r=1$, but stronger when $1<r<q$. (One can also check that the only SCIs that are also grille inequalities are the 3 -cycle inequalities.)

The following theorem characterises the facet-defining SCIs.

Theorem 3 The strengthened circulant inequality (11) defines a facet of $P(m, n)$ if and only if (i) $p=q+1$ (i.e., the inequality is a simple circulant inequality) or (ii) $q+1<p \leq 3 q / 2$ and $p<m$.

Proof. See Appendix C.

We remark that the condition $q+1<p \leq 3 q / 2$ implies that $q \geq 4$ and $p \geq 6$. It also implies that $r>1$, i.e., the SCI is not combinatorial.

\subsection{On $(p, q)$ inequalities}

Now we consider again the $(p, q)$ inequalities (7). An obvious necessary condition for a $(p, q)$ inequality to define a facet is that $\Phi_{G}(w+1)=p$ and $\Phi_{G}(w)=p-1$ (since, otherwise, the inequality would be dominated by a strong HI). Unfortunately, checking whether these two equations hold is easily shown to be $\mathcal{N} \mathcal{P}$-hard (e.g., by reduction from perfect matching in uniform hypergraphs [13].) The main goal of this subsection is to show that, when $q=2$, it is possible to check in polynomial time whether a given $(p, q)$ inequality is facet-defining.

When $q=2$, each node in $I^{\prime}$ is adjacent to exactly 2 nodes in $J^{\prime}$. We will find it helpful to define a reduced graph, denoted by $G^{-}$. The vertex set of $G^{-}$is $J^{\prime}$, and the edge set, denoted by $E^{-}$, is defined as follows. There is an edge $\{u, v\}$ in $E^{-}$if and only if there exists some $i \in I^{\prime}$ such that the edges $\{i, u\}$ and $\{i, v\}$ exist in $E$. This construction is illustrated in Figure 4. The graph $G$ on the left corresponds to a $(p, 2)$ inequality with $I^{\prime}=\left\{s_{1}, \ldots, s_{6}\right\}$ and $J^{\prime}=\left\{t_{1}, \ldots, t_{5}\right\}$. The large and small circles represent facilities in $I^{\prime}$ and clients in $J^{\prime}$, respectively, and the lines represent the edges in $E$. The inequality is:

$$
x\left(s_{1}, t_{1}\right)+x\left(s_{1}, t_{2}\right)+\cdots+x\left(s_{6}, t_{4}\right)+x\left(s_{6}, t_{5}\right) \leq 2+\sum_{i=1}^{6} y\left(s_{i}\right) .
$$



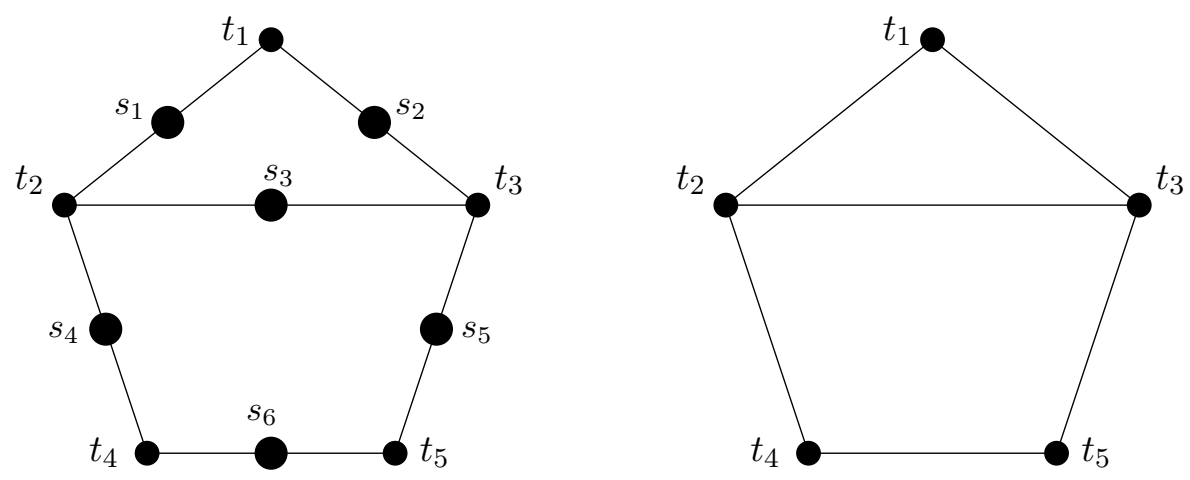

Figure 4: Graph $G$ (left) and associated reduced graph $G^{-}$(right).

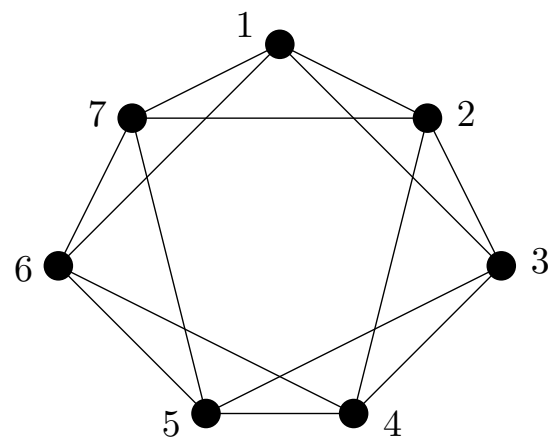

Figure 5: A super-matchable graph on 7 nodes.

The corresponding reduced graph $G^{-}$is shown on the right. So, for example, the edge $\left\{t_{1}, t_{2}\right\}$ is present in $E^{-}$because the edges $\left\{s_{1}, t_{1}\right\}$ and $\left\{s_{1}, t_{2}\right\}$ exist in $E$.

We now define a certain property that $G^{-}$may or may not have:

Definition 3 The reduced graph $G^{-}=\left(J^{\prime}, E^{-}\right)$is said to be super-matchable if, for every edge $\{u, v\} \in E^{-}$and every node $w \in J^{\prime} \backslash\{u, v\}$, there is a perfect matching of the nodes in $J^{\prime} \backslash\{u, v, w\}$.

So, for example, the reduced graph $G^{-}$shown on the right of Figure 4 is not supermatchable, because, if we take the edge $\left\{t_{1}, t_{2}\right\}$ and the node $t_{5}$, the nodes $t_{3}$ and $t_{4}$ cannot be matched. On the other hand, the graph shown in Figure 5 is super-matchable. For example, if we take the edge $\{1,3\}$ and the node 5 , the remaining nodes, $2,4,6$ and 7 , can be matched using the edges $\{2,4\}$ and $\{6,7\}$.

Note that one can check whether a given graph $G^{-}$is super-matchable in polynomial time. The property of being super-matchable turns out to be 
crucial for determining whether a $(p, 2)$ inequality defines a facet.

Theorem $4 A(p, 2)$ inequality defines a facet of $P(m, n)$ if and only if the associated reduced graph $G^{-}$is super-matchable.

Proof. From the result in [6] on lifting, mentioned in Subsect. 2.3, it suffices to show that a $(p, 2)$ inequality can be lifted if and only if $G^{-}$is super-matchable. So, consider an edge $\{u, v\}$ in $G^{-}$, and let $i$ be a facility in $I^{\prime}$ such that the edges $\{i, u\}$ and $\{i, v\}$ are in $G$. (Note that there may be more than one such facility.) Consider the lifted inequality that is obtained by changing the coefficient of $x_{i w}$ from zero to one, for some $w \in J^{\prime} \backslash\{u, v\}$. To see whether this lifted inequality is valid, we attempt to construct a feasible SPLP solution that violates it, in which $x_{i w}$ takes the value 1 . Note that, in such a solution, $y_{i}$ must take the value 1 as well. Then, to maximise the left-hand side of the lifted inequality, it pays to set $x_{i u}$ and $x_{i v}$ to one as well. Now we have a contribution of 3 to the left-hand side and 1 to the right-hand side. Then, the only way the lifted inequality could be violated would be for the remaining $p-3$ clients in $T$ to be served by only $(p-3) / 2$ of the remaining facilities in $I^{\prime}$. This can happen if and only if those $p-3$ clients can be matched in $G^{-}$.

To complete the proof, it suffices to show that it is not possible to lift $x_{i j}$ when $i \in I \backslash I^{\prime}$ and/or $j \in J \backslash J^{\prime}$. To see this, just note that any extreme point of $P(m, n)$ that satisfies the $(p, 2)$ inequality at equality and has $x_{i j}=y_{i}=1$ violates the lifted inequality.

The following proposition presents an exponentially large family of supermatchable graphs, which corresponds to an exponentially large family of new facet-defining $(p, 2)$ inequalities.

Proposition 8 Let $p \geq 5$ be an odd integer and let $k$ be an integer with $2 \leq k \leq\lfloor p / 2\rfloor$. Let $G(p, k)$ be the graph with vertex set $\{1, \ldots, p\}$, and an edge between nodes $i$ and $j$ if and only if $|i-j| \bmod p \leq k$. (The graph in Figure 5 is $G(7,2)$.) Then $G(p, k)$ is super-matchable.

Proof. One can check that, if one removes any three nodes from such a graph, the remaining nodes can be matched.

We remark that the graphs $G(p, k)$ are a special case of the so-called antiwebs, defined by Trotter [22] in the context of set packing. (Note however that the associated $(p, 2)$ inequalities are not related in any simple way to Trotter's antiweb inequalities, since we are talking about the reduced graph $G^{-}$, not $G$ itself.)

To close this section, we briefly examine the general case, in which $q$ may be larger than 2 . The following proposition gives a simple (and polynomialtime checkable) necessary condition for a $(p, q)$ inequality to define a facet. 


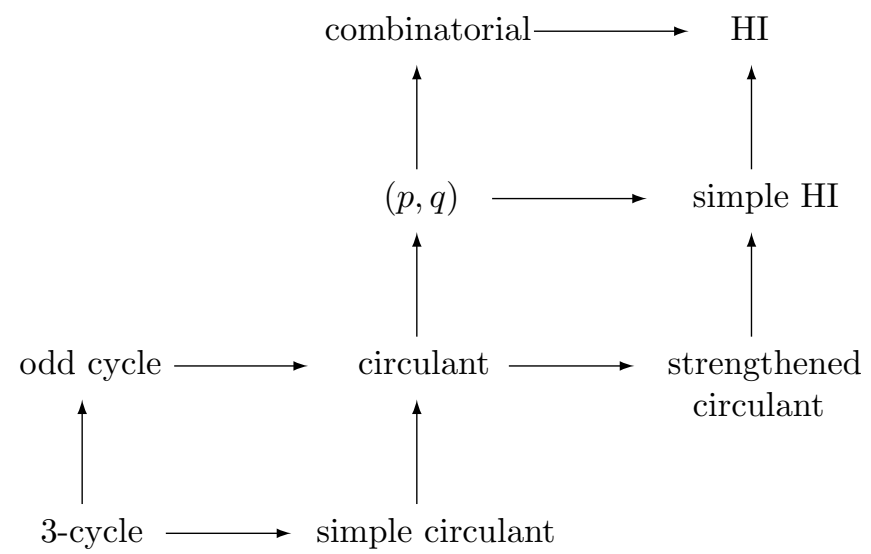

Figure 6: Hierarchy of inequalities for the SPLP.

Proposition 9 If a $(p, q)$ inequality is facet-defining, then $\Phi_{G}^{+}(w+1)=p$, $\Phi_{G}^{+}(w)=p-1$ and $r=1$.

Proof. If either of the first two conditions does not hold, then the $(p, q)$ inequality is dominated by a simple HI. Now observe that, since each node in $I^{\prime}$ has degree $q$ in $G$, we have $\Phi_{G}^{+}(w) \leq w q=p-r$. So, if the second condition holds, $r$ must be 1 .

The next proposition shows that there are many non-trivial facet-defining $(p, q)$ inequalities with $r=1$.

Proposition 10 For all $p$ and $q$ with $2 \leq q<p$ and $r=1$, there exist exponentially many facet-defining $(p, q)$ inequalities that are not simple circulant inequalities.

Proof. When $r=1$, the facet-defining simple HIs described in Theorem 2 reduce to $(p, q)$ inequalities.

To aid the reader, we display in Figure 6 a hierarchy of nine families of valid inequalities. An arrow from one class to another means that the latter is a proper generalisation of, or dominates, the former. One can see that the HIs subsume the other inequalities in the figure.

\section{Facility Augmentation}

In this section, we present and analyse a new technique, called facility augmentation, that enables one to derive new valid inequalities from known ones. We will see that, when applied to facet-defining HIs, it can yield 
facet-defining inequalities that are not HIs (and not equivalent to known inequalities).

The following theorem describes the technique in its full generality.

Theorem 5 Let $\alpha^{T} x \leq \beta^{T} y+\gamma$ be a valid inequality for $P(m, n)$, with $\alpha$, $\beta, \gamma$ non-negative, and let $S$ be an arbitrary subset of $I$. For each $j \in J$, let $\alpha^{+}(S, j)=\max _{i \in S}\left\{\alpha_{i j}\right\}$. The "augmented" inequality

$$
\sum_{i \in I} \sum_{j \in J} \alpha_{i j} x_{i j}+\sum_{j \in J} \alpha^{+}(S, j) x_{m+1, j} \leq \sum_{i \in I} \beta_{i} y_{i}+\left(\sum_{i \in S} \beta_{i}\right) y_{m+1}+\gamma
$$

is valid for $P(m+1, n)$.

Proof. Suppose that there exists an extreme point of $P(m+1, n)$, say $(\bar{x}, \bar{y})$, that violates the augmented inequality. We must have $\bar{y}_{m+1}=1$, since, if $\bar{y}_{m+1}=0$, the VUBs force the second term on the left-hand side to be zero, and the augmented inequality reduces to the original inequality. Now suppose that $\bar{x}_{i j}=1$ for some $i \in S$ and $j \in J$. Then we get an extreme point that violates the augmented inequality by at least as much by changing $\bar{x}_{i j}$ to 0 and changing $\bar{x}_{m+1, j}$ to 1 instead. So we can assume that $\bar{x}_{i j}=0$ for all $i \in S$ and $j \in J$. Then, if $\bar{y}_{i}=1$ for any $i \in S$, we can get an extreme point that violates the augmented inequality by even more by changing $\bar{y}_{i}$ to 0 .

In summary, we can assume that $\bar{y}_{m+1}=1$ and $\bar{y}_{i}=0$ for all $i \in S$. Now, we can get another extreme point that violates the augmented inequality by the same amount by (a) closing facility $m+1$, (b) opening all of the facilities in $S$, and (c) taking each client that was assigned to facility $m+1$ and reassigning it to the facility in $S$ that maximises $\alpha_{i j}$. This extreme point violates the original inequality, which contradicts the fact that the original inequality was valid.

We remark that, when $|S|=1$, facility augmentation reduces to the "facility replication" procedure described in Theorem 3.3. of Cho et al. [6].

Facility augmentation can of course be applied more than once if desired. Specialising the technique to the case in which the original valid inequality is homogeneous, we obtain the following corollary.

Corollary 2 Let $G=\left(I^{\prime}, J^{\prime}, E\right)$ be a connected bipartite graph, and let $x(E) \leq \beta y\left(I^{\prime}\right)+\gamma$ be an HI. For each $i \in I^{\prime}$, let $n(i)$ denote the set of neighbours of $i$ in $G$. Let $T \subseteq I \backslash I^{\prime}$ be an arbitrary set of facilities, and, for each $i \in T$, let $S(i)$ be an arbitrary subset of $I^{\prime}$. Take $G$ and construct a larger graph, $G^{+}$, as follows. For each facility $i \in T$, we add a node to $I^{\prime}$. For each facility $i \in T$ and each client $j \in \bigcup_{i^{\prime} \in S(i)} n\left(i^{\prime}\right)$, we add the edge 
$\{i, j\}$ to $E$. Let $E^{+}$denote the resulting set of edges. Then the following (not necessarily homogeneous) inequality is valid:

$$
x\left(E^{+}\right) \leq \beta y\left(I^{\prime}\right)+\beta \sum_{i \in T}|S(i)| y_{i}+\gamma .
$$

Proof. For a fixed $i \in T$, apply Theorem 5 with $S$ set to $S(i)$, and renumber the facilities so that facility $m+1$ becomes facility $i$. Repeat the argument for each facility in $T$.

We call the inequalities (12) augmented HIs. A natural question is to determine conditions for augmented HIs to define facets. The following proposition gives some necessary conditions.

Proposition 11 Suppose the augmented HI (12) defines a facet. Then:

1. For each facility $i \in T$, the degree of node $i$ in $G^{+}$is greater than $\beta|S(i)|$.

2. There does not exist a set $\tilde{S} \subset I^{\prime}$ such that $|\tilde{S}|<|S(i)|$ and $\bigcup_{i^{\prime} \in \tilde{S}} n\left(i^{\prime}\right) \subseteq$ $\bigcup_{i^{\prime} \in S(i)} n\left(i^{\prime}\right)$.

3. There does not exist a set $\tilde{S} \subset I^{\prime}$ such that $|\tilde{S}|=|S(i)|$ and $\bigcup_{i^{\prime} \in \tilde{S}} n\left(i^{\prime}\right)$ is a proper subset of $\bigcup_{i^{\prime} \in S(i)} n\left(i^{\prime}\right)$.

Proof. If the first condition does not hold for some $i \in T$, then we can obtain a stronger augmented HI by removing node $i$ and its incident edges from $G^{+}$. (The original augmented HI is implied by the new one and the VUBs for the removed edges.) If the second condition does not hold, we can obtain a stronger augmented HI by replacing $S(i)$ with a suitable set $\tilde{S}$. (The set of edges $E^{+}$either stays the same or increases, and the coefficient of $y_{i}$ decreases.) The same applies if the third condition does not hold. (The coefficient of $y_{i}$ stays the same, and the set of edges $E^{+}$increases.)

Unfortunately, checking the second condition in Proposition 11 is $\mathcal{N} \mathcal{P}$ hard (by reduction from set cover.) We conjecture that determining whether an augmented $\mathrm{HI}$ defines a facet is also $\mathcal{N} \mathcal{P}$-hard. On the positive side, the following theorem shows that there exist facet-defining augmented simple HIs that are not HIs.

Theorem 6 Suppose we apply facility augmentation to the facet-defining simple HIs described in Theorem 2. Suppose that, for each $i \in T$, the sets $n\left(i^{\prime}\right)$ for $i^{\prime} \in S(i)$ are disjoint. Then the augmented inequality is facetdefining. 
Proof. Let $T$ denote the set of added facilities, and let $t=|T|$. From Theorem 2, the simple HI associated with the original graph $G$ defines a facet of $P(m, n)$. Also, any root of the original simple HI can be converted into a root of the augmented version simply by closing all of the facilities in $T$. Now, the dimension of $P(m+t, n)$ is $t(n+1)$ more than that of $P(m, n)$, since we have added $t$ new $y$ variables and $t n$ new $x$ variables. So, it suffices to construct $n+1$ new affinely-independent roots of the augmented inequality for each $t \in T$.

Let $i \in T$ be fixed. One of the desired roots can be obtained as follows. Take a root of the original inequality in which all facilities in $S(i)$ are open and, for all $i^{\prime} \in S(i)$, every client in $n\left(i^{\prime}\right)$ is assigned to facility $i^{\prime}$. Convert this into a root of the augmented inequality by (a) opening facility $i$, (b) closing the facilities in $T \cup S(i) \backslash\{i\}$, and (c) re-assigning all clients that were assigned to a facility in $S(i)$ to facility $i$.

Now we construct an additional $|n(i)|$ roots for the given $i \in T$. For each client $j \in n(i)$, we construct a root by opening facility $i$, assigning the clients in $n(i) \backslash\{j\}$ to facility $i$, and opening a subset of the facilities in $I \backslash(S(i) \cup\{i\})$, chosen in such a way that each of the clients in $(J \backslash n(i)) \cup\{j\}$ can be assigned to one of them.

Finally, we need to construct an additional $J-|n(i)|$ roots for the given $i$. For each client $j \in J \backslash n(i)$, we construct a root by opening facility $i$ and assigning all of the clients in $n(i) \cup\{j\}$ to it, then opening a subset of the facilities in $I \backslash(S(i) \cup\{i\})$, chosen in such a way that each of the clients in $J \backslash(n(i) \cup\{j\})$ can be assigned to one of them.

Here is an example. Recall that the graph $G$ shown in Figure 3 corresponds to a simple HI that defines a facet of $P(m, n)$ for $m \geq 10$ and $n \geq 5$. Add one new facility node, say $s_{1234}$, which is adjacent to client nodes $t_{1}$ to $t_{4}$. The resulting graph $G^{+}$is shown in Figure 7 . (The labels of the ten original facility nodes have been omitted, for clarity.) The augmented simple HI corresponding to the resulting graph defines a facet of $P(m+1, n)$.

We also have the following corollary.

Corollary 3 For all $p$ and $q$ with $2 \leq q<p$ and $p \bmod q=1$, there exist facet-defining augmented $(p, q)$ inequalities that are not $(p, q)$ inequalities.

Proof. When $p \bmod q=1$, the facet-defining augmented simple HIs described in Theorem 6 are augmented $(p, q)$ inequalities.

Again, the number of facet-defining inequalities described in both Theorem 6 and Corollary 3 grows exponentially with both $m$ and $n$.

\section{Conclusion}

In this paper, we presented the homogeneous inequalities for the SPLP, which include many previously-known inequalities as special cases. Al- 


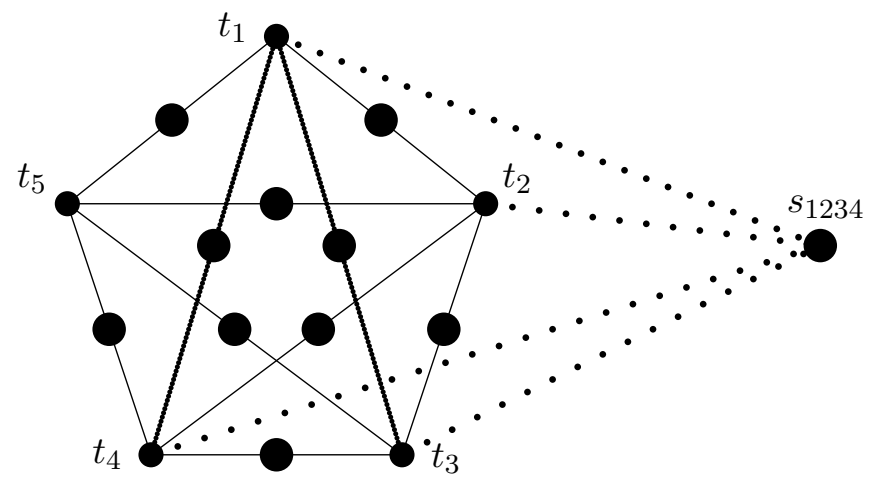

Figure 7: Representation of a facet-defining augmented simple HI inequality.

though computing the best possible coefficients for a homogeneous inequality appears to be difficult, we derived some useful conditions for them to define facets. We also derived some conditions for the $(p, q)$ inequalities, which are a very special case of the homogeneous inequalities, to define facets. Finally, we presented a new lifting procedure, and showed that it enables one to derive still more facet-defining inequalities.

An obvious topic for future research is the design of effective (exact or heuristic) separation algorithms for the inequalities described in this paper. In fact, we believe that there is also a need to design improved separation algorithms for some of the other inequalities in the literature, such as those described in $[1,3,4,5,6,11]$. Such algorithms are likely to be essential if one wishes to solve very hard SPLP instances, such as the ones in $[14,16,18]$.

\section{References}

[1] Aardal, K.: Capacitated facility location: separation algorithms and computational experience. Math. Program., 81, 149-175 (1998)

[2] Balinski, M.: Integer programming: methods, uses, computation. Mgmt. Sci., 12, 254-313 (1965)

[3] Cánovas, L., Landete, M., Marín, A.: New facets for the set packing polytope. Oper. Res. Lett., 27, 153-161 (2000)

[4] Cánovas, L., Landete, M., Marín, A.: On the facets of the simple plant location packing polytope. Discr. Appl. Math., 124, 27-53 (2002)

[5] Cho, D.C., Johnson, E.J., Padberg, M.W., Rao, M.R.: On the uncapacitated location problem I: valid inequalities and facets. Math. Oper. Res., 8, 579-589 (1983a) 
[6] Cho, D.C., Johnson, E.J., Padberg, M.W., Rao, M.R.: On the uncapacitated location problem II: facets and lifting theorems. Math. Oper. Res., 8, 590-612 (1983b)

[7] Chvátal, V.: On certain polytopes associated with graphs. J. Combin. Th. B, 18, 138-154 (1975)

[8] Conforti, M., Cornuéjols, G., Zambelli, G.: Integer Programming. Graduate Texts in Mathematics, vol. 271. Springer, Berlin (2015)

[9] Cornuéjols, G., Fischer, M.L., Nemhauser, G.L.: On the uncapacitated location problem. Ann. Discr. Math., 1, 163-177 (1977)

[10] Cornuéjols, G., Nemhauser, G.L., Wolsey, L.A.: The uncapacitated facility location problem. In: Mirchandani, P.B., Francis, R.L. (eds.) Discrete Location Theory, pp. 1-54. Wiley, New York (1990)

[11] Cornuéjols, G., Thizy, J.-M.: Some facets of the simple plant location polytope. Math. Program., 23, 50-74 (1982)

[12] Fernández, E., Landete, M.: Fixed-charge location problems. In G. Laporte et al. (eds.) Location Science, pp. 47-77. Springer, Cham (2015)

[13] Garey, M., Johnson, D.S.: Computers and Intractability. Freeman, San Francisco (1979)

[14] Ghosh, D.: Neighborhood search heuristics for the uncapacitated facility location problem. Eur. J. Oper. Res., 150, 150-162 (2003)

[15] Guignard, M.: Fractional vertices, cuts and facets of the simple plant location problem. Math. Program. Study, 12, 150-162 (1980)

[16] Kochetov, Y., Ivanenko, D.: Computationally difficult instances for the uncapacitated facility location problem. In T. Ibaraki et al. (eds) Metaheuristics: Progress as Real Problem Solvers, pp. 351-367. Springer, Berlin (2005)

[17] Korte, B., Vygen, J.: Combinatorial Optimization: Theory and Algorithms (5th Edn.) Algorithms and Combinatorics, vol. 21. Springer, Berlin (2012)

[18] Kratica, J., Tosic, D., Filipovic, V., Ljubic, I.: Solving the simple plant location problem by genetic algorithm. RAIRO Oper. Res., 35, 127-142.

[19] Labbé, M., Louveaux, F.: Location problems. In: Dell'Amico, M., Maffoli, F., Martello, S. (eds.) Annotated Bibliographies in Combinatorial Optimization, pp. 261-281. Wiley, Chichester (1997) 


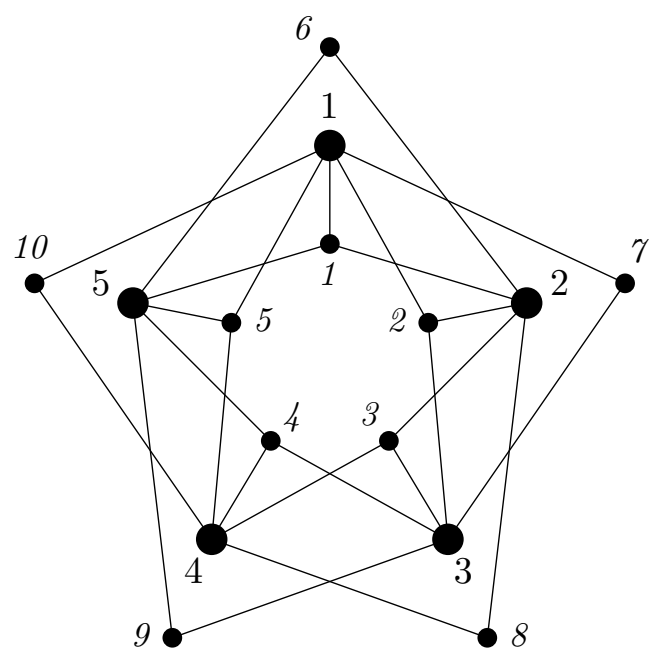

Figure 8: Graph $G$ used in the proof of Theorem 1.

[20] Letchford, A.N., Miller, S.J.: An aggressive reduction scheme for the simple plant location problem. Eur. J. Oper. Res., 234, 674-682 (2014)

[21] Posta, M., Ferland, J., Michelon, P.: An exact cooperative method for the uncapacitated facility location problem. Math. Program. Comput., $6,199-231(2014)$

[22] Trotter, L.E.: A class of facet-producing graphs for vertex packing polyhedra. Discr. Math., 12, 373-388 (1975)

[23] Verter, V.: Uncapacitated and capacitated facility location problems. In: Eiselt, H.A., Marianov, V. (eds.) Foundations of Location Analysis, pp. 25-38. International Series in Operations Research \& Management Science, vol. 155. Springer, Berlin (2011)

\section{Appendix A: Proof of Theorem 1}

Let $m=5$ and $n=10$, and let $G$ be the graph displayed in Figure 8 . (Numbers in italics indicate clients.) One can check that $\kappa(G)=3, \Phi_{G}(1)=$ $5, \Phi_{G}(2)=8$ and $\Phi_{G}(3)=10$. Thus, $\Delta_{G}(2)=3$ and $\Delta_{G}(3)=2$. Setting $k=$ 2 , we obtain the strong HI $x(E) \leq 3 y(I)+2$. This HI is not combinatorial, since it has $\beta>1$, and it is not a grille inequality, since it has $\gamma>1$. Moreover, we have $k=2<\kappa(G)=3$.

To complete the proof, we have to show that the HI defines a facet of $P(5,10)$. We partition $E$ into two subsets:

$$
E^{1}=\{\{i, j\} \in E: j \leq 5\} \text { and } E^{2}=\{\{i, j\} \in E: j \geq 6\} .
$$


We will call an extreme point of $P(5,10)$ a "root" of the HI if it satisfies it at equality. Once can check that there are three kinds of roots:

1. $y(I)=1, x\left(E^{1}\right)=3, x\left(E^{2}\right)=2$;

2. $y(I)=2, x\left(E^{1}\right)=4, x\left(E^{2}\right)=4$;

3. $y(I)=2, x\left(E^{1}\right)=5, x\left(E^{2}\right)=3$.

Now, let $\alpha^{T} x+\beta^{T} y=\gamma$ be an equation that is satisfied by all roots. One root of the second kind is obtained by opening facilities 1 and 2, assigning clients $1,2,4,5,7,9$ and 10 to facility 1 , and assigning the other clients to facility 2 . If we modify this root by re-assigning client 1 to facility 2 , we obtain another root. This shows that $\alpha_{11}=\alpha_{21}$. The same argument shows that $\alpha_{1 j}=\alpha_{2 j}$ for $j \in\{2,4,9\}$.

One root of the third kind is obtained by opening facilities 1 and 3 , assigning clients $1,2,5,6,7,8$ and 10 to facility 1 , and assigning clients 3,4 and 9 to facility 3 . If we modify this root by re-assigning client 2 to facility 3 , we obtain another root. This shows that $\alpha_{12}=\alpha_{32}$. The same argument shows that $\alpha_{1 j}=\alpha_{3 j}$ for $j \in\{6,7,8\}$.

By symmetry, we must have:

$$
\begin{array}{ll}
\alpha_{i j}=\alpha_{k j} & \left(j \in J,\{i, j\},\{k, j\} \in E^{1}\right) \\
\alpha_{i j}=\alpha_{k j} & \left(j \in J,\{i, j\},\{k, j\} \in E^{2}\right) \\
\alpha_{i j}=\alpha_{k j} & (j \in J,\{i, j\},\{k, j\} \notin E) .
\end{array}
$$

Moreover, due to the assignment constraints (2), we can assume that $\alpha_{i j}=0$ for all $\{i, j\} \notin E$.

So far, we have established that the equation $\alpha^{T} x+\beta^{T} y=\gamma$ must take the form

$$
\sum_{j \in J} \alpha_{j} \sum_{i:\{i, j\} \in E} x_{i j}+\sum_{i \in I} \beta_{i} y_{i}+\gamma
$$


Now, consider the following square matrix of order 15 :

$$
\left(\begin{array}{lllllllllllllll}
1 & 0 & 0 & 0 & 0 & 1 & 1 & 0 & 0 & 1 & 0 & 1 & 0 & 0 & 1 \\
0 & 1 & 0 & 0 & 0 & 1 & 1 & 1 & 0 & 0 & 1 & 0 & 1 & 0 & 0 \\
0 & 0 & 1 & 0 & 0 & 0 & 1 & 1 & 1 & 0 & 0 & 1 & 0 & 1 & 0 \\
0 & 0 & 0 & 1 & 0 & 0 & 0 & 1 & 1 & 1 & 0 & 0 & 1 & 0 & 1 \\
0 & 0 & 0 & 0 & 1 & 1 & 0 & 0 & 1 & 1 & 1 & 0 & 0 & 1 & 0 \\
1 & 1 & 0 & 0 & 0 & 1 & 1 & 1 & 0 & 1 & 1 & 1 & 1 & 0 & 1 \\
0 & 1 & 1 & 0 & 0 & 1 & 1 & 1 & 1 & 0 & 1 & 1 & 1 & 1 & 0 \\
0 & 0 & 1 & 1 & 0 & 0 & 1 & 1 & 1 & 1 & 0 & 1 & 1 & 1 & 1 \\
0 & 0 & 0 & 1 & 1 & 1 & 0 & 1 & 1 & 1 & 1 & 0 & 1 & 1 & 1 \\
1 & 0 & 0 & 0 & 1 & 1 & 1 & 0 & 1 & 1 & 1 & 1 & 0 & 1 & 1 \\
1 & 0 & 1 & 0 & 0 & 1 & 1 & 1 & 1 & 1 & 0 & 1 & 0 & 1 & 1 \\
0 & 1 & 0 & 1 & 0 & 1 & 1 & 1 & 1 & 1 & 1 & 0 & 1 & 0 & 1 \\
0 & 0 & 1 & 0 & 1 & 1 & 1 & 1 & 1 & 1 & 1 & 1 & 0 & 1 & 0 \\
1 & 0 & 0 & 1 & 0 & 1 & 1 & 1 & 1 & 1 & 0 & 1 & 1 & 0 & 1 \\
0 & 1 & 0 & 0 & 1 & 1 & 1 & 1 & 1 & 1 & 1 & 0 & 1 & 1 & 0
\end{array}\right) .
$$

The first 5 columns represent $y_{1}, \ldots, y_{5}$. The other 10 columns represent the quantity $\sum_{i:\{i, j\} \in E} x_{i j}$ for $j=1, \ldots, 10$. One can check that each of the rows represents a root. (There are 5 roots of the first kind, 5 of the second, and 5 of the third.) Using a linear algebra package, one can check that the only equation satisfied by all 15 rows is (up to scaling by a constant) $x(E)=3 y(I)+2$. Thus, the HI is facet-defining.

\section{Appendix B: Proof of Theorem 2}

Let us write $p=w q+r$, where $r$ is the remainder when dividing $p$ by $q$. As in Appendix A, we call an extreme point of $P(m, n)$ a "root" of the simple $\mathrm{HI}$ if it satisfies it at equality, and let $\alpha^{T} x+\beta^{T} y=\gamma$ be an equation that is satisfied by all roots. One can check that there are two kinds of roots. One kind has $x(E)=p-r$ and $y(S)=w$, and the other has $x(E)=p$ and $y(T)=w+1$.

Consider any $j \in J \backslash T$ and any root (of either kind) such that client $j$ is assigned to an open facility in $S$. We can obtain another root (of the same kind) by assigning client $j$ to any other open facility. By symmetry, this shows that $\alpha_{i j}$ takes the same value for all $i \in I$. Due to the assignment constraints (2), we can assume that this value is zero.

Next, consider any $i \in I \backslash S$, and any root (of either kind) such that facility $i$ is closed. By opening that facility, we obtain another root (of the same kind). By symmetry, this shows that $\beta_{i}=0$ for all $i \in I \backslash S$.

Next, consider any $j \in T$, and any root of the first kind such that client $j$ is assigned to a facility in $I \backslash S$. By assigning $j$ to any other open facility $i$ such that $\{i, j\} \notin E$, we obtain another root. By symmetry, this shows 
that $\alpha_{i j}$ takes the same value for all $i \in I$ such that $\{i, j\} \notin E$. Due to the assignment constraints (2), we can assume that this value is zero.

So far, we have shown that the equation $\alpha^{T} x+\beta^{T} y=\gamma$ must take the form

$$
\sum_{\{i, j\} \in E} \alpha_{i j} x_{i j}=\sum_{i \in S} \beta_{i} y_{i}+\gamma
$$

Now consider a root of the second kind. There must exist a facility $i \in S$ that has fewer than $q$ clients in $T$ assigned to it. Accordingly, there must exist a client $j \in T$ that is currently assigned to a facility in $S \backslash\{i\}$, but for which $\{i, j\} \in E$. We can obtain another root of the second kind by assigning that client to $i$ instead. By symmetry, this shows that, for any given client $j \in T$, the coefficient $\alpha_{i j}$ takes the same value for all $i$ such that $\{i, j\} \in E$.

Now, consider any pair $j, j^{\prime} \in T$, and let $i \in S$ be such that both $\{i, j\}$ and $\left\{i, j^{\prime}\right\}$ belong to $E$. Consider any root of the first kind such that facility $i$ is open, client $j$ is assigned to facility $i$, and client $j^{\prime}$ is assigned to an open facility in $I \backslash S$. We obtain another root by assigning client $j^{\prime}$ to facility $i$ and assigning client $j$ to the open facility in $I \backslash S$. By symmetry, this shows that $\alpha_{i j}$ takes the same value for all $\{i, j\} \in E$.

We have now shown that the equation $\alpha^{T} x+\beta^{T} y=\gamma$ must take the form

$$
\alpha_{0} \sum_{\{i, j\} \in E} x_{i j}=\sum_{i \in S} \beta_{i} y_{i}+\gamma,
$$

where $\alpha_{0}$ is a scalar. Now, for any $i \in S$, consider a root of the second kind such that facility $i$ is open and exactly $r$ clients are assigned to $i$. By closing facility $i$, and assigning each of those $r$ clients to an open facility in $I \backslash\{i\}$, we obtain another root. This shows that $\beta_{i}=r \alpha_{0}$. Therefore, the equation $\alpha^{T} x+\beta^{T} y=\gamma$ must take the form:

$$
\alpha_{0} \sum_{\{i, j\} \in E} x_{i j}=r \alpha_{0} \sum_{i \in S} y_{i}+\gamma .
$$

Now, if $\alpha_{0}$ were equal to zero, $\gamma$ would have to be zero, and the equation would become vacuous. So $\alpha_{0} \neq 0$. Dividing the equation by $\alpha_{0}$, we obtain:

$$
\sum_{\{i, j\} \in E} x_{i j}=r \sum_{i \in S} y_{i}+\gamma
$$

and the only possible value for $\gamma$ is the one given in the simple HI.

\section{Appendix C: Proof of Theorem 3}

As in Appendix B, we let $p=w q+r$, and we call an extreme point of $P(m, n)$ a "root" of the SCI if it satisfies it at equality. We also let $S=\left\{s_{1}, \ldots, s_{p}\right\}$ 
and $T=\left\{t_{1}, \ldots, t_{p}\right\}$. One can check that the roots of an SCI are of two kinds:

- $x(E)=p$ and $y(S)=w+1$,

- $x(E)=p-r$ and $y(S)=w$.

Moreover, the special structure of the graph $G$ associated with the SCI implies that, for a root of the second kind, the open facilities are "spread out", in the sense that $\left|(i \bmod p)-\left(i^{\prime} \bmod p\right)\right| \geq q$ for all pairs $i, i^{\prime}$ of open facilities.

One can check the following facts. When $p>2 q$, all roots (of either kind) satisfy the equation $x\left(s_{i}, t_{i+q+r}\right)=0$ for $i=1, \ldots, p$. When $3 q / 2<p<2 q$ and $q$ is odd, all roots satisfy the equation $x\left(s_{i}, t_{i+(q-1) / 2}\right)=y\left(s_{i}\right)$ for $i=$ $1, \ldots, p$. When $3 q / 2<p<2 q$ and $q$ is even, all roots satisfy the equations $x\left(s_{i}, t_{i+q / 2-1}\right)=x\left(s_{i}, t_{i+q / 2}\right)=y\left(s_{i}\right)$ for $i=1, \ldots, p$. When $q+2 \leq p \leq 3 q / 2$ and $p=m$, all roots satisfy the equation $x\left(s_{i}, t_{i+q}\right)=x\left(s_{i}, t_{i+q+1}\right)$ for $i=1, \ldots, p$. So the SCI cannot define a facet in any of these cases.

Since we know from [15] that the simple circulant inequalities define facets, all that remains is to show that the SCIs define facets when condition (ii) holds. As usual, let $\alpha^{T} x+\beta^{T} y=\gamma$ be an equation that is satisfied by all roots.

Since we assume that $p<m, S$ is a proper subset of $I$. Given any facility $i \in I \backslash S$, there exists a root (of either kind) such that facility $i$ is closed. By opening that facility, we obtain another root. So $\beta_{i}=0$ for all $i \in I \backslash S$.

Now let $i$ be an arbitrary facility in $I \backslash S$. We obtain a root of the first kind by opening facilities $s_{1}$ and $i$, assigning clients $t_{1}, \ldots, t_{q}$ to facility $s_{1}$, and assigning all other clients to facility $i$. Now let $j$ be any client in $J \backslash\left\{t_{1}, \ldots, t_{q}\right\}$. We obtain another root by re-assigning client $j$ to facility $i$. This shows that $\alpha_{1 j}=\alpha_{i j}$. By symmetry, this implies that

$$
\alpha_{i j}=\alpha_{k j} \quad(j \in J,\{i, j\},\{k, j\} \notin E) .
$$

Moreover, due to the assignment constraints (2), we can assume that $\alpha_{i j}=0$ for all $\{i, j\} \notin E$.

Now, for $i=r+1, \ldots, q$, we obtain a root of the second kind by opening facilities 1 and $i$, assigning clients $t_{1}, \ldots, t_{q}$ to facility 1 , and assigning the other clients to facility $i$. If we modify this root by re-assigning client $t_{q}$ to facility $i$, we obtain another root. This shows that $\alpha\left(1, s_{q}\right)=\alpha\left(i, s_{q}\right)$ for $i=r+1, \ldots, q$. By symmetry, this implies that

$$
\alpha_{i j}=\alpha_{k j} \quad\left(j \in\left\{t_{1}, \ldots, t_{p}\right\},\{i, j\},\{k, j\} \in E\right) .
$$

So far, we have shown that the equation $\alpha^{T} x+\beta^{T} y=\gamma$ must take the form

$$
\sum_{j \in T} \alpha_{j} \sum_{\{i, j\} \in E} x_{i j}=\sum_{i \in S} \beta_{i} y_{i}+\gamma .
$$


Now, for $i=r+1, \ldots, p-r+1$, we obtain a root of the second kind by opening facilities 1 and $i$, assigning clients $t_{1}, \ldots, t_{q}$ to facility 1 , and assigning the other clients to facility $i$. A comparison of these roots shows that $\beta_{i}$ must take the same value for $i=r+1, \ldots, p-r+1$. By symmetry, this implies that all of the $\beta_{i}$ are equal. So our equation takes the form:

$$
\sum_{j \in S} \alpha_{j} \sum_{\{i, j\} \in E} x_{i j}=\beta y(S)+\gamma .
$$

Now, for $i=1, \ldots, p$, we obtain a root of the first kind by opening facility $s_{i}$ and assigning all clients to it. A comparison of these roots shows that $\alpha_{j}$ must take the same value for all $j \in T$. So our equation reduces to $\alpha x(E)=\beta y(S)+\gamma$, and the proof is complete. 